\title{
A Penetrating Look at Ice Friction
}

\section{A new approach for studying friction on ice helps explain why the ease of sliding depends strongly on temperature, contact pressure, and speed.}

\author{
By Kārlis Agris Gross
}

$$
\text { a }
$$
n Latvia, where bobsledding, luge, and skeleton are popular, ice can be more than just "slippery." The local language has another term slidamība-roughly translated as "slideability" - which refers to the ease of movement on a surface. This terminology signifies the awareness that sliding on ice depends on multiple factors-something physicists have had trouble explaining despite 160 years of effort. Previous work has focused on the water layer that forms between the ice surface and the sliding object, say, an ice skate. However, this model does not show why friction is higher near the ice melting point than it is at lower temperatures (Fig. 1). A new study of the solid properties of ice may provide a solution. Rinse Liefferink from the University of Amsterdam and colleagues have performed a series of experiments, in which they measure both the friction of a sliding object and the hardness of ice over a wide range of conditions [1]. The observations show that the hardness decreases as the temperature increases, leading to a high-friction "ploughing" behavior once the sliding object is able to penetrate the softer ice surface. This novel approach to studying ice friction could help in developing technologies that improve safety for winter drivers or give an edge to winter athletes.

Previous work has related slipperiness to a surface layer (or film) of water. The thickness of this water layer could explain how friction varies with temperature [2]. At very cold temperatures (around $-100^{\circ} \mathrm{C}$ ), melting is minimal, and the surface is considered dry-providing a possible explanation for the large observed friction. As temperatures warm to an intermediate range (around $-20^{\circ} \mathrm{C}$ ), the developing water layer acts as a thin lubricating film that could explain the observed decrease in friction. However, it becomes less clear what happens near the melting point at $0^{\circ} \mathrm{C}$, where the observed friction increases again. The models explain this reduced slipperiness as arising from a thicker film of water, but observations have not been able to confirm this [3].

In previous studies of ice friction, researchers have had difficulty in controlling all the relevant parameters, such as temperature and surface smoothness. Normally, such tribology experiments have been performed on a small-scale rheometer, in which a rotating probe is pressed down on an ice disk. One problem with these setups is that the rotating probe, or "slider," often follows a single track and thereby moves repetitively over disturbed ice.

Liefferink and colleagues used a rheometer at low loads with three different slider shapes: a big sphere, a small sphere, and a model skate. The team kept the ice smooth by repeatedly adding a fresh film of water to the surface, and they varied the ice temperatures over a wide range from $-120{ }^{\circ} \mathrm{C}$ to $-1.5^{\circ} \mathrm{C}$. In addition to measuring friction with the different sliders, the researchers measured the ice hardness using a high-load mechanical testing machine that presses on the ice with a spherical probe. The value of the hardness is given by the force needed to penetrate or indent the surface with the probe.

The data showed how ice hardness varied with temperature and sliding speed. The ice became harder at colder temperatures (Fig. 1), while at a given temperature, the hardness increased at faster indentation and sliding speeds. The hardness behavior helped the team interpret the ice friction observations. At the lowest temperatures, the large sliding friction was attributed to water molecules being held rigidly on 


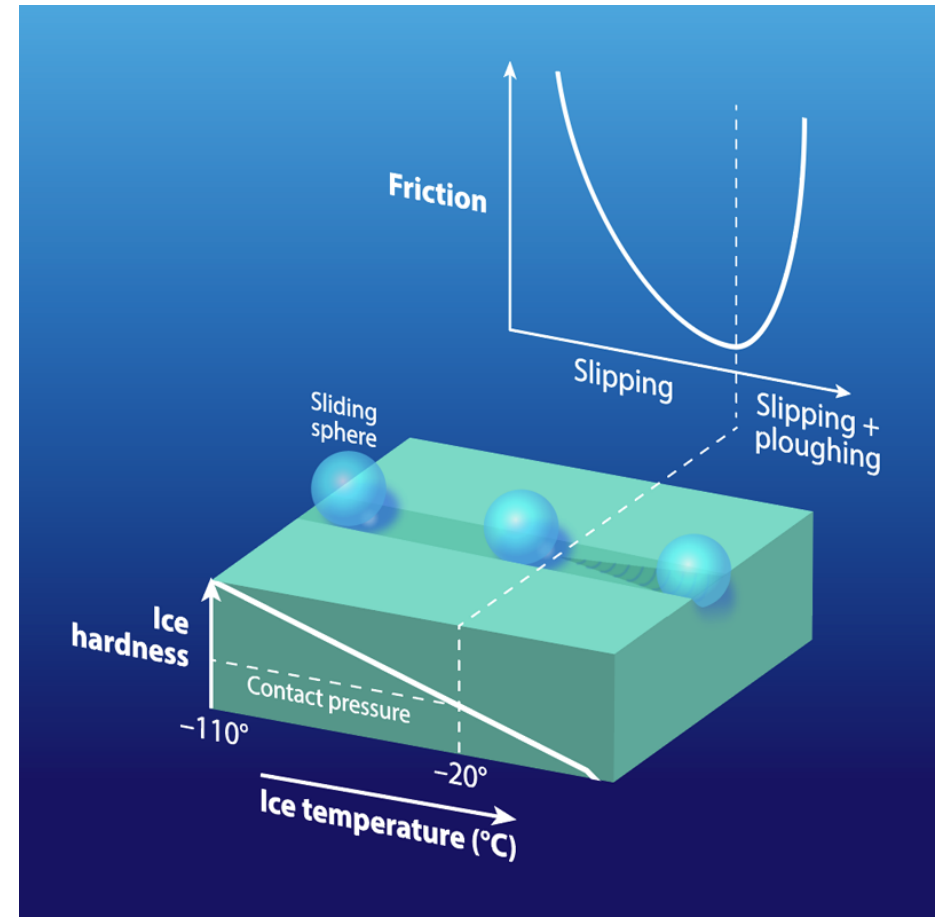

Figure 1: This graphic shows how the hardness of ice decreases with increasing temperature. A sphere moving across the surface will penetrate the surface when the ice hardness is below the sphere's contact pressure. In this case, the sphere will plough over the surface, experiencing more and more friction as the temperature increases and the hardness drops.

Credit: APS/Alan Stonebraker

the surface. These molecules were made more mobile by the shearing action of the slider, and this mobility became greater-and the friction lower-at intermediate temperatures. However, at higher temperatures, the friction increased because of the decrease in hardness that allowed the slider to plough into the ice. Despite this drop in hardness, we know that ice remains sufficiently hard for sliding at temperatures close to the melting point. Most other materials become soft-and "unslidable"-near their melting point.

Different slider shapes started to plough at different temperatures. At a given pressing force, the small sphere showed ploughing at about $-20^{\circ} \mathrm{C}$, but the larger skate-section showed ploughing at $-8^{\circ} \mathrm{C}$. This behavior is explained by a lower contact pressure from the skate's larger size (larger contact area). Besides delaying the onset of ploughing, the

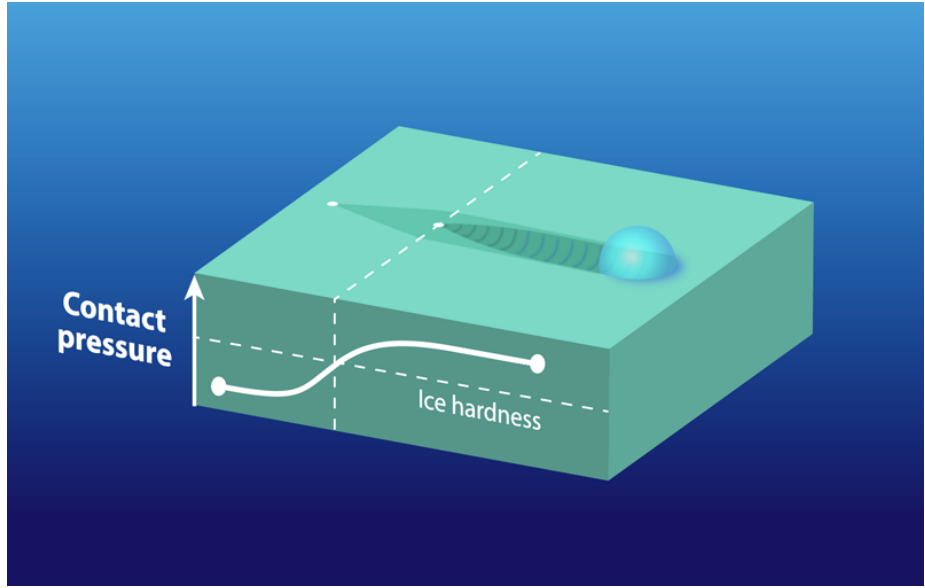

Figure 2: A sudden increase in contact pressure can occur when, for example, a bobsled team jumps onto their sled. The friction increases as more of the slider comes into contact with the ice. If the contact pressure becomes larger than the ice hardness, the slider will plough into the surface, increasing the friction significantly.

Credit: APS/Alan Stonebraker

lower contact pressure of the skate provides more mobility to surface water molecules, making the skate slide better than the sphere even at colder temperatures. Friction's dependence on contact pressure is mediated by how much of the slider's surface is in contact with the ice and whether the pressure is enough to initiate ploughing (Fig. 2). Experiments at low speeds showed that ice hardness increases with slider speed, implying that a fast skate should plough less and thus slide better.

Further work should investigate how ice friction and slideability are affected by weather conditions, faster sliding speeds, and ice structure. Weather is an issue because it can affect the water layer thickness. My team has recently performed experiments with a skeleton sled and showed that humidity, air temperature, and ice temperature jointly influence the sliding speed [4]. We have also looked at the effect of slider surface topography and loading on sliding speed [5]. To measure the effect at faster sliding conditions, researchers will need an appropriate test facility, such as the bobsled ice track in Sigulda, Latvia, where higher speeds can be attained in a long, straight section [6]. Such tests will need an advanced sensor system to accurately and simultaneously measure both ice friction and air drag [7]. 
The last element for further study is how ice's structure helps regulate the hardness. Ice is known to self-heal after surface scratches. It may turn out that ice is like steel in that it becomes harder after being mechanically stressed, or "cold worked." Materials science needs to offer advanced characterization and testing under extreme conditions to completely unravel the slideability of ice.

Kārlis Agris Gross: Biomaterials Research Group, Faculty of Materials Science and Applied Chemistry, Riga Technical University, Riga, Latvia

\section{REFERENCES}

1. R. W. Liefferink et al., "Friction on ice: How temperature, pressure, and speed control the slipperiness of ice," Phys. Rev. X 11, 011025 (2021).

2. A.-M. Kietzig et al., "Physics of ice friction," J. Appl. Phys. 107,
081101 (2010).

3. T. Bartels-Rausch et al., "A review of air-ice chemical and physical interactions ( $\mathrm{AICl}$ ): liquids, quasi-liquids, and solids in snow," Atmos. Chem. Phys. 14, 1587 (2014).

4. E. Jansons et al., "Influence of weather conditions on sliding over ice at a push-start training facility," Biotribology 25, 100152 (2021).

5. E. Jansons et al., "Measurement of sliding velocity on ice, as a function of temperature, runner load and roughness, in a skeleton push-start facility," Cold Reg. Sci. Technol. 151, 260 (2018).

6. International Bobsleigh and Skeleton Federation list of 16 global ice-tracks, https://www.ibsf.org/en/tracks.

7. M. Irbe et al., "Unveiling ice friction and aerodynamic drag at the initial stage of sliding on ice: Faster sliding in winter sports," Tribol. Int. (to be published). 